\title{
Characterizing solution dynamics of highly flexible enzymes
}

\author{
Maxwell B. Watkins ${ }^{1}$, Steve P. Meisburger ${ }^{1}$ and Nozomi Ando ${ }^{1,2}$
}

1. Department of Chemistry, Princeton University

2. Department of Chemistry \& Chemical Biology, Cornell University

\begin{abstract}
One of the most challenging aspects of modern structural biology is characterizing highly flexible protein systems. Often, high-resolution structural techniques such as X-ray crystallography or, more recently, cryo-electron microscopy, may be insufficient to fully describe the wide range of motions important to the function of these systems. The use of chromatography-coupled small-angle X-ray scattering (SAXS) along with mathematical component separation techniques such as singular value decomposition (SVD) and evolving factor analysis (EFA) can yield high-quality SAXS data on highly dynamic, complex systems. The use of these techniques enables us to model these systems with higher confidence, helping to elucidate the nature of more complex conformational mixtures.
\end{abstract}

